\title{
La pandemia de COVID-19 en Colombia y los desafíos de la respuesta sanitaria
}

DOI: https://doi.org/10.11144/Javeriana.rgps20.covid

\author{
Amparo Hernández-Bello ${ }^{a}$ \\ Pontificia Universidad Javeriana, Colombia \\ ORCID: https://orcid.org/0000-0002-4831-6755 \\ Sandra Milena Agudelo-Londoño \\ Pontificia Universidad Javeriana, Colombia \\ ORCID: https://orcid.org/0000-0002-8980-6590
}

\begin{abstract}
Para citar esta presentación Hernández-Bello A, Agudelo-Londoño SM. La pandemia de COVID-19 y los desafíos de la respuesta sanitaria. Rev Gerenc Polit Salud. 2021;20. https://doi.org/10.11144/ Javeriana.rgps20.covid
\end{abstract}

a Autora de correspondencia: ahernand@javeriana.edu.co 
En marzo de 2020, la Organización Mundial de la Salud (OMS) declaró que el brote del nuevo coronavirus detectado en diciembre de 2019 en la República Popular China (2019-nCoV) era una pandemia, tanto por la extensión geográfica del contagio (al momento más de 140 países) como por la gravedad de sus efectos en términos del número de personas infectadas y de pérdida de vidas humanas (1). La enfermedad por coronavirus denominada COVID-19 (por sus siglas en inglés) es la primera pandemia por coronavirus de la que se tiene registro.

La pandemia de COVID-19 no es solo un problema sanitario, es esencialmente un problema social complejo de grandes magnitudes. Como lo señala Ignacio Ramonet (2), se trata de un "hecho social total" por su dimensión global y porque ha afectado todos los espacios de la vida: social, económico, político y cultural como lo conocemos hasta hoy.

Esta experiencia mundial inédita, de más de dieciocho meses de crisis sistémica, ha revelado el carácter socialmente determinado de la pandemia y cómo el comportamiento de la afectación y deterioro de la salud y condiciones de vida, que recae duramente sobre los individuos y las poblaciones más pobres y vulneradas, es síntoma de las profundas desigualdades sociales y sanitarias existentes. Al decir de Saskia Sassen, se puede pensar la pandemia “...como una oportunidad para entender más y mejor las injusticias de nuestro sistema. Esto no es nuevo, pero creo que en el contexto de este virus hay algo que se puede visibilizar mejor" (3). Es decir, la crisis no es el virus, lo que está en crisis es nuestro modelo civilizatorio actual, y es allí donde debiéramos centrar nuestra atención.

Enfrentar las causas estructurales de la pobreza y de las inequidades sociales asociadas a esta crisis es un desafío principal de las políticas y las instituciones, porque de estas dependen el comportamiento y control de la pandemia pero, sobre todo, el futuro de nuestras vidas. Un reto más grande aún en regiones y sociedades muy desiguales en las que buena parte de la población sigue excluida de los beneficios de la protección social y, en particular, del acceso universal a la atención sanitaria. Esta es la situación de América Latina y el Caribe —la región más desigual del planeta- sobre la que la pandemia y sus efectos han recaído con particular fuerza, habida cuenta de fenómenos como la pobreza, la informalidad, la inseguridad alimentaria y las deficiencias estructurales de los sistemas de salud y los mecanismos de protección social. Esta situación la resume la Comisión Económica para América Latina y el Caribe -CEPAL y la Organización Panamericana de la Salud-OPS en un informe conjunto de 2020, de la siguiente manera:

El COVID-19 llega a una región marcada por una matriz de desigualdad social, cuyos ejes estructurantes - el estrato socioeconómico, el género, la etapa del ciclo de vida, la condición etnico-racial, el territorio, la situación de discapacidad y el estatus migratorio, entre otros - generan escenarios de exclusión y discriminación múltiple y simultánea, que redundan en una mayor vulnerabilidad ante la enfermedad. Estas desigualdades también se expresan en el ámbito de la salud, tanto en la cobertura, el acceso efectivo y los resultados de los servicios de salud, como en las condiciones basales de salud de las personas y las comunidades. Por ende, es clave considerar esta desigualdad en la respuesta a la pandemia. (4, p.11). 
Responder a la pandemia en este escenario de inequidades y vulnerabilidades preexistentes, con sistemas de salud fragmentados, segmentados, desfinanciados, de rectoría débil e insuficientes en sus funciones de salud pública es un desafío mayor. Y si bien la respuesta no se reduce a las decisiones del sector de la salud, hay mucho que aprender de lo que esta crisis revela sobre la centralidad del bienestar que debe ser la protección de la salud y la vida y el papel de los sistemas de salud en su defensa.

Si algo sabemos hoy de esta experiencia es que hay naciones con sistemas de salud que han transitado mejor la crisis y dado mejor respuesta tanto a las necesidades de prevención y control de la pandemia, como al mantenimiento de la atención de las demás condiciones agudas y crónicas prevalentes. Se trata de sistemas públicos, integrales, fortalecidos en su infraestructura y financiamiento para la operación (5), que han hecho de la Atención Primaria de Salud integral e integrada y su abordaje territorial, comunitario y familiar, participativo e intersectorial, orientado a mejorar la situación de los grupos más desaventajados y excluidos de la sociedad, la principal estrategia de atención (6).

En síntesis, para afrontar esta y otras emergencias sanitarias es necesario fortalecer en lo inmediato los sistemas de salud para lidiar con la enfermedad, el sufrimiento y la agonía que genera la pandemia y así transitar hacia sistemas universales que permitan enfrentar sus efectos y los efectos del impacto económico que definirá también la situación de la salud futura de la población. Hasta ahora las decisiones de política han sido reactivas más que preventivas en relación con la epidemia y han primado las decisiones económicas sobre las sanitarias. Por ello, como bien dice Vicenç Navarro (7), no debemos caer en la falsa dicotomía que sugiere que hacer prioritarias las intervenciones para controlar y superar la pandemia atenta contra la necesaria recuperación económica, porque no habrá recuperación económica si no se reducen el alcance de la pandemia y sus efectos en pérdida de vidas humanas.

Nuestra Revista Gerencia y Políticas de Salud quiere atender el reto de reflexionar, discutir y proponer sobre estos asuntos, en consonancia con su misión de publicar artículos originales que contribuyan a la comprensión y análisis de temas estructurales y coyunturales sobre la salud de la población y sus determinantes, la configuración de los sistemas de salud y las racionalidades políticas y gerenciales de la respuesta organizada a los problemas de salud. Con ese propósito, el presente dossier temático busca completar el panorama de los análisis biomédicos sobre la pandemia de los que se ocupan numerosas publicaciones académicas, estimulando otras lecturas a partir de cuestionamientos sobre lo que la pandemia ha evidenciado acerca de las inequidades en materia de salud y bienestar de las poblaciones; de las vulnerabilidades tradicionalmente existentes y las emergentes; de la capacidad y naturaleza de los sistemas y servicios de salud para responder a la emergencia, y de las consecuencias de la toma de decisiones sobre la saludenfermedad, el bienestar de la población y los sistemas y servicios de salud. 
Hemos compilado ocho artículos originales de investigación realizados entre el segundo semestre de 2020 e inicios de 2021, escrito por autoras y autores pertenecientes a universidades, centros de investigación e instituciones del sector salud, quienes desde distintos enfoques y aproximaciones metodológicas acogieron la convocatoria para responder a una o varias de las preguntas sobre los retos sociales y sanitarios de la pandemia de COVID-19. Algunos trabajos abordan las necesidades y demandas de poblaciones y/o regiones de especial interés y vulneración durante la crisis, otros las características de la respuesta sectorial para responder a la emergencia sanitaria o los desafíos para superarla, y unos más se adentran en el desarrollo de herramientas sencillas y cercanas para el análisis de datos o discuten estrategias para el rastreo de contactos de cara a los derechos ciudadanos.

En el artículo "Medidas para la mitigación de la pandemia del COVID-19 en la salud de las niñas, los niños y adolescentes en contextos de protección: una síntesis de recomendaciones de política en América, 2020", investigadoras e investigadores de las universidades de Antioquia y San Buenaventura analizan las recomendaciones para la protección y mitigación de los efectos de la pandemia por COVID-19 en la salud física y mental de niñas, niños y adolescentes de diversos programas de restablecimiento de derechos en los sistemas de protección del continente americano. Concluyen que las condiciones excepcionales de la pandemia se convierten en una ventana de oportunidad política para retomar las discusiones sobre modalidades alternativas de restablecimiento de derechos diferentes al internamiento en instituciones cerradas, sobre la capacitación de las y los cuidadores y sobre la necesidad de propiciar espacios de participación de las niñas, niños y adolescentes en las decisiones que influyen en su bienestar.

En el marco del Observatorio Ruralidad y Paz en contextos de pandemia y postpandemia, se desarrolló la investigación "Salud colectiva en tiempos de COVID-19. Modelo escalar para comprender las afectaciones en la ruralidad de Urabá, Antioquia, Colombia, 2020" de Beatriz Elena Arias López, Eulalia Hernández Ciro y Alderid Gutiérrez Loaiza, de la Universidad de Antioquia. Las autoras identifican las dinámicas de integración/exclusión relacionadas con la salud colectiva en los territorios rurales de la subregión del Urabá antioqueño en el contexto de la pandemia COVID-19, y llegan a la conclusión que existen grandes desbalances sociales estructurales, especialmente en las zonas rurales de Colombia, los cuales implican limitaciones para una gobernanza efectiva de la salud en tiempos de crisis.

Dos contribuciones se adentran en el análisis de la red hospitalaria pública y su papel en el manejo de la pandemia. La primera es la investigación titulada "El estado de la red pública hospitalaria en Colombia para enfrentar el COVID-19, posterior a la política de categorización de riesgo fiscal y financiero" de Gilma Stella Vargas Peña, de la Universidad de Antioquia; Laura Estefany Ruiz Sánchez, de la red pública de salud de Medellín, Metrosalud, y Luis Alberto Martínez Saldarriaga, director de la Asociación de Empresas Sociales del Estado de Antioquia. En esta se discute cómo las políticas de transformación y reestructuración de los hospitales públicos implementadas en los últimos años, desde lógicas neoliberales y basadas en los modelos de la Nueva Gestión Pública, han limitado la cantidad, el crecimiento y la capacidad resolutiva de la red pública hospitalaria nacional, afectando la respuesta ante el COVID-19. 
La segunda contribución, "Gestión centralizada de camas de Unidad de Cuidado Intensivo e Intermedio por el Centro Regulador de Urgencias y Emergencias de Bogotá ante el COVID-19" es autoría de Andrés José Álvarez Villegas, Ángela María Roa Saldaña y Edizabeth Ramírez Rodríguez, de la Dirección de Urgencias y Emergencias en Salud de la Secretaría Distrital de Salud de Bogotá, la red pública hospitalaria más grande del país. En esta se presentan los resultados del análisis de la estrategia de coordinación centralizada de camas en las Unidades de Cuidados Intensivos e Intermedios que el Distrito Capital emprendió en el marco del aumento de casos de COVID-19 en la ciudad, y se concluye que esta ha resultado ser una estrategia efectiva que favorece la prestación de servicios de salud especializados a pacientes en estado crítico.

Completa este panorama sobre la respuesta del sistema, el texto "El desinterés en recibir la vacuna contra el coronavirus en población de 23 ciudades colombianas, julio a diciembre de 2020" de Pablo Enrique Chaparro Narváez y Carlos Andrés Castañeda Orjuela, integrantes del Observatorio Nacional de Salud del Instituto Nacional de Salud. En su investigación los autores encontraron que la principal razón de la población para no recibir la vacuna, es la creencia que esta puede ser insegura por sus potenciales efectos adversos, y esperan que sus hallazgos informen a las autoridades de salud sobre la importancia de diseñar estrategias educativas y comunicacionales que permitan aumentar la confianza de las comunidades en la eficacia y seguridad de la vacunación contra COVID-19.

Sumado a las contribuciones anteriores, desde el Instituto de Salud Pública de la Pontificia Universidad Javeriana en Bogotá, Sandra Milena Agudelo Londoño y Clara Andrea Núñez Avilés proponen revisar la "Discusión en redes sociales sobre el rastreo digital de contactos para COVID-19: retos para la salud pública desde Colombia". A través de una etnografía para Internet que caracterizó las aplicaciones móviles creadas en Colombia para el rastreo digital de contactos, se analizó la discusión ciudadana en las redes sociales sobre las implicaciones de esta práctica en salud pública, considerando elementos relacionados con la privacidad y la seguridad informática de los datos de las personas, la transparencia y cuidado en la obtención y uso subsecuente de la información como la posible mercantilización de los datos, las implicaciones del rastreo más allá de lo sanitario, en especial, los riesgos de una vigilancia ciudadana, la afectación a las libertades individuales y los límites de un gobierno de la vida a través de lo digital.

Cierra este dossier el trabajo "Caracterización de la tendencia del COVID-19 en Colombia con regresiones polinomiales en Excel" del profesor Ariel Emilio Cortés Martínez, de la Universidad Javeriana Bogotá, y Carmen Elisa Becerra Huertas, de la Universidad Colegio Mayor de Cundinamarca, con una propuesta metodológica para el seguimiento del crecimiento y tendencia de la curva de contagios y muertes por COVID-19 en Colombia a través de modelos polinomiales, como una estrategia de fácil construcción para la vigilancia de eventos de salud pública que puede facilitar la toma de decisiones oportunas. 
Esperamos que la lectura de estos materiales contribuya a la discusión informada, a la reflexión que oriente mejor la toma de decisiones y que, también, convoque la acción colectiva en defensa de la salud y la vida. Queda abierto este espacio de diálogo permanente con el que nuestra revista está comprometida. Sus contribuciones serán siempre bienvenidas.

\section{Referencias}

1. Organización Mundial de la Salud. Alocución de apertura del Director General de la OMS en la rueda de prensa sobre la COVID-19 celebrada el 11 de marzo de 2020. 2020. Disponible en: https://www.who.int/es/director-general/speeches/detail/who-director-general-s-opening-remark s-at-the-media-briefing-on-covid-19---11-march-2020.

2. Ramonet I. La pandemia y el sistema-mundo. Un hecho social total. Comunicación. Estudios venezolanos de comunicación. 2020, 190-191:95-124.

3. Keve C, Sassen S. Entrevistas Dipló. Podemos pensar esta pandemia como una oportunidad para entender más y mejor las injusticias de nuestro sistema. Entrevista a Saskia Sassen por Carolina Keve. Le Monde Diplomatic, edición Cono Sur. Disponible en: https://www.eldiplo.org/notas-web/podemos-pensar-esta-pandemia-como-una-oportunidad-para -entender-mas-y-mejor-las-injusticias-de-nuestro-sistema/

4. Cepal-OPS. Salud y economía: una convergencia necesaria para enfrentar el COVID-19 y retomar la senda hacia el desarrollo sostenible en América Latina y el Caribe. Informe COVID-19, 30 de julio de 2020. 2020. Disponible en: https://www.eldiplo.org/notas-web/podemos-pensar-esta-pandemia-c omo-una-oportunidad-para-entender-mas-y-mejor-las-injusticias-de-nuestro-sistema/

5. Laurell AC. Prólogo. En: Tetelboin C, Iturrieta D, Schor-Landman C, Coords. América Latina. Sociedad, política y salud en tiempos de pandemia. Ciudad Autónoma de Buenos Aires: CLACSO; Xochimilco: Universidad Autónoma Metropolitana; Xalapa: Universidad Veracruzana; Cochabamba: Universidad Mayor de San Simón; Valparaíso: Universidad de Valparaíso; 2021. 11-16.

6. Giovanella L, et al. ¿Es la Atención Primaria de Salud Integral parte de la respuesta a la pandemia de Covid-19 en Latinoamérica? Trabalho, Educação e Saúde. 2021; 19. Disponible en: https://www.sc ielo.br/j/tes/a/CJX9Rs5gSBJmsMrfwhkdJrL/

7. Navarro V. Una falsa dicotomía: salvar la salud de la población o facilitar la recuperación económica. En: Pandemia, economía y Estado del bienestar; 2020. 120-8. 